\title{
Stage IIA Bone Cancer AJCC v8
}

National Cancer Institute

\section{Source}

National Cancer Institute. Stage IIA Bone Cancer A/CC v8. NCI Thesaurus. Code C136617.

Stage IIA includes: T1, NO, M0, G2 or G3. T1: Tumor measuring $8 \mathrm{~cm}$ or less in greatest dimension. N0: No regional lymph node metastasis. M0: No distant metastasis. G2:

Moderately differentiated, high grade. G3: Poorly differentiated, high grade. (AJCC 8th ed.) 